\title{
Baseline Shift in Neuronal Oscillations and Its Implications for the Interpretation of Evoked Activity Obtained with EEG/MEG
}

\author{
Alina A. Studenova ${ }^{1 *}$, Arno Villringer ${ }^{2,3}$, Vadim V. Nikulin ${ }^{2,4,5}$ \\ ${ }^{1}$ School of Psychology, Faculty of Social Sciences, National Research University \\ Higher School of Economics, Moscow, Russia \\ 2 Neurology Department, Max Planck Institute for Human Cognitive and Brain \\ Sciences, Leipzig, Germany \\ ${ }^{3}$ Department of Cognitive Neurology, University Hospital Leipzig, Leipzig, Germany \\ ${ }^{4}$ Centre for Cognition and Decision Making, Institute for Cognitive Neuroscience, \\ National Research University Higher School of Economics, Moscow, Russia \\ ${ }^{5}$ Neurophysics Group, Department of Neurology, Charité-Universitätsmedizin Berlin, \\ Berlin, Germany \\ * Corresponding author, astudenova@hse.ru
}

\begin{abstract}
Oscillations and evoked responses are two types of neuronal activity recorded non-invasively with EEG/MEG. Although typically studied separately, they might in fact represent the same process. One possibility to unite them is to demonstrate that neuronal oscillations have nonzero mean which would indicate that stimulus-relating amplitude modulation of neuronal oscillations should lead to the generation of evoked responses. We validated this mechanism using computational modelling and analysis of a large EEG data set. With a biophysical model generating alpha rhythm, we indeed demonstrated that the oscillatory mean is nonzero for a large range of model-parameter values. In EEG data we detected non-zero mean alpha oscillations in about $96 \%$ of the participants. Furthermore, using neuronal-ensemble modelling, we provided an explanation for the often observed discrepancies between amplitude modulation and baseline shifts. Overall, our results provide strong support for the unification of neuronal oscillations and evoked responses.
\end{abstract}

\section{Introduction}

Neuronal oscillations represent one of the main forms of neuronal activity in the human brain and they have been implicated in diverse sensory, motor and cognitive functions (Buzsáki and Draguhn, 2004). Another prominent form of neuronal activity is evoked responses (evoked potentials, evoked fields) which are transient phase-locked events produced by stimuli, movement and cognitive operations. These two phenomena are usually studied separately but there are two general ways through which they can be interrelated. On the one hand, ongoing neuronal oscillations can modulate evoked responses (Becker et al., 2008, 
Roberts et al., 2014, Iemi et al., 2019, Stephani et al., 2020). These modulations are probably due to the fact that neuronal oscillations, such as alpha and beta rhythms, are associated with changes in cortical excitability which in turn affect the recruitment of neurons responding to stimuli. On the other hand, evoked responses may in fact represent changes in certain aspects of neuronal oscillations from which it would follow that both phenomena should not be treated separately.

Three mechanisms of evoked responses have been proposed over several decades: the additive mechanism (Wood and Allison, 1981, Shah et al., 2004, Mäkinen et al., 2005, Mazaheri and Jensen, 2006), the phase resetting mechanism (Savers et al., 1974, Makeig et al., 2002, Hanslmayr et al., 2007, Rawls et al., 2020), and the baseline-shift mechanism (BSM; Nikulin et al., 2007, Mazaheri and Jensen, 2008, Nikulin et al., 2010, Iemi et al., 2019). The additive mechanism states that evoked responses are generated independently of the ongoing oscillations, that is to say, "in addition to" (i.e. evoked response is a stand-alone phenomenon). The phase reset mechanism suggests that after a stimulus presentation phases of ongoing oscillators switch to a particular value (i.e., evoked responses are oscillations). Finally, BSM implies that any amplitude modulation of ongoing oscillations with a non-zero oscillatory mean (OM) leads to the generation of evoked responses (i.e. posits that evoked responses and oscillations reflect the same process). There has been a considerable debate in the literature on whether auditory (Shah et al., 2004, Mäkinen et al., 2005, Ahmadi et al., 2021), visual (Makeig et al., 2002, Mazaheri and Jensen, 2006, Hanslmayr et al., 2007, Iemi et al., 2019, Rawls et al., 2020, Ahmadi et al., 2021), and somatosensory (Nikulin et al., 2007) evoked responses can be generated through the additive, phase reset mechanism, or BSM and currently, in the EEG/MEG community, there is no clear cut evidence in strong support of either of mechanisms. It should be noted that these mechanisms are not assumed to be mutually exclusive and may co-exist. Moreover, they may play a role at different latencies of evoked response generation and in different conditions.

The third mechanism - BSM - is based on two prerequisites: 1) sensory stimuli or movements should modulate the amplitude of ongoing oscillations (these findings being confirmed in almost all EEG/MEG studies on oscillations); 2) neuronal oscillations have a non-zero OM. A combination of these two aspects should unavoidably lead to the generation of an evoked response. BSM gives rise to the following predictions: 1) post-stimulus oscillations are accompanied by amplitude modulation, 2) non-zero OM sign and direction of amplitude modulation (increase or decrease) determine the polarity of an evoked response, 3) time and spatial distribution of modulation correlate with both the evoked response time course and spatial topography.

Consistent with the predictions, the presence of non-zero OM has been previously directly confirmed (Nikulin et al., 2007, Nikulin et al., 2010) and the generation of evoked responses has been found to be consistent with the idea of BSM (Mazaheri and Jensen, 2008, Iemi et al., 2019. Iemi et al., 2021). Moreover, many empirical studies assessed cognitive functioning with simultaneous analysis of evoked responses and oscillations, with the results showing consistent correlation of evoked response amplitude and/or latency with the modulation in oscillations (Yordanova et al., 2001, Funderud et al., 2012, Abeles and Gomez-Ramirez, 2014, Störmer et al., 2016, Schneider and Maguire, 2018). However, several other studies observed little or no correlation (Fukuda et al., 2015, Xia et al., 2020). Therefore, factors that might have caused conflicting results should be considered and clarified.

The intuition about oscillations having non-zero OM stems from asymmetrical morphological and biophysical properties of neurons and neuronal networks that affect the generation of oscillations in a way that should create a non-zero OM activity. These asymmetries emerge 
on several scales: a membrane, a cell, and external inputs. On the membrane level, inward and outward currents flow through synapses and voltage- and ligand-activated channels that are placed asymmetrically along the neuron (Bekkers, 2000, Benhassine and Berger, 2005. Bannister, 2005, Boivin and Nedivi, 2018). On the cellular level, the contribution of various dendrites to a current dipole is not equal and depends on the orientation of a process with respect to the longitudinal axis of the apical dendrite (Schomer and Da Silva, 2010, Neymotin et al., 2020). On the exogenous inputs level, proximal and distal drives arrive at different layers of the neocortex, thus creating an asymmetrical redistribution of charges (Douglas and Martin, 2004, Spruston, 2008). Overall, based on our current knowledge about neuronal currents, it is highly unlikely that currents flowing towards soma will have the same magnitude as outward dendritic currents (Nikulin et al., 2007, Mazaheri and Jensen, 2008). However, previous research on BSM was focused on EEG/MEG findings and, at the moment, there is no modelling study providing further evidence for the presence of a non-zero OM.

Despite that, there is no strict necessity to measure OM directly. If neuronal oscillations have a non-zero OM, any modulation of oscillations' amplitude affects the mean as well, thus leading to a baseline shift in lower frequency. Consequently, on the macroscopic recordings, there would be an association between low-frequency time course and the amplitude envelope of ongoing oscillations. Therefore, in the case of BSM, amplitude modulation bears a significant role both for observation of evoked responses and for empirical estimation of OM. However, the prominence of amplitude modulation may change. Therefore, as previous research sampled data exclusively from young adults and only during either eyes-closed or eyes-open state, and the sample size was relatively small, validation on the bigger data set with both eyes-closed and eyes-open conditions and with diverse age groups is necessary.

In the present study, we aimed to verify the prediction about asymmetric currents leading to non-zero OM with the use of a biophysically realistic computational model. We examined a model of a small cortical patch that generates alpha oscillations - Human Neocortical Neurosolver (HNN; Neymotin et al., 2020). To understand how OM can be affected by diverse changes in the model on the level of a single neuron, we varied several biophysical and morphological properties of neurons in the network, such as voltage-gated channel densities, length of dendrites, and intensity of incoming stimuli. Next, we attempted to validate BSM further on the large data set including young and elderly participants with eyes-open and eyes-closed sessions. Finally, we elaborated on the factors that might mask a correspondence between the amplitude modulation of oscillations and corresponding baseline shifts. For this, we investigated the effects of spatial synchronization within a network with population modelling using simple oscillators (Schaworonkow and Nikulin, 2019).

\section{Results}

\section{Asymmetric currents lead to non-zero mean oscillations}

The simulations with Human Neocortical Neurosolver (HNN; Neymotin et al., 2020) demonstrated that a biologically plausible model of a population of neurons in the neocortex generated alpha frequency oscillations centred around a non-zero oscillatory mean (OM). In HNN, alpha oscillations are generated by rhythmic inputs (proximal and distal) that arrive in the network in an alternating fashion. The main output of HNN is a current dipole estimate that is computed as the sum of the intracellular currents within the pyramidal neurons' dendrites projected onto a direction parallel to the apical dendrite plane. Note that currents that are flowing down are associated with a negative sign, and upward currents - with a positive 
sign (Neymotin et al., 2020). To provide evidence for non-zero mean alpha oscillations, we systematically varied parameters in the model (Figure 1a) and examined associated changes in OM that were driven by changes in currents (Figure 1 $1 \mathrm{~b}$ ).

As an extreme case, we simulated alpha rhythm with either proximal or distal input. When only the proximal drive was active, a strong current was flowing from basal dendrites to soma and from soma to apical dendrite. This upward current produced a positive current dipole (Figure 2a), with OM being persistently above zero. On the opposite, when only distal input activated the network, currents flew from the top of the apical dendrite in the downward direction (Figure 20.). These dynamics created a shift in OM such that it became less than zero. In both cases, alpha rhythm was preserved. An example of the alpha rhythm simulated with both proximal and distal input using default settings is displayed in Figure 2 $\mathrm{b}$. The $\mathrm{OM}$ for this case is above zero. The simulations with isolated proximal and isolated distal input represent an exceptional case and serve to demonstrate the effect of proximal and distal drives on dendritic currents and consequently on OM. The other presented results can be conceptualized as intermediate stages between these two extremes.
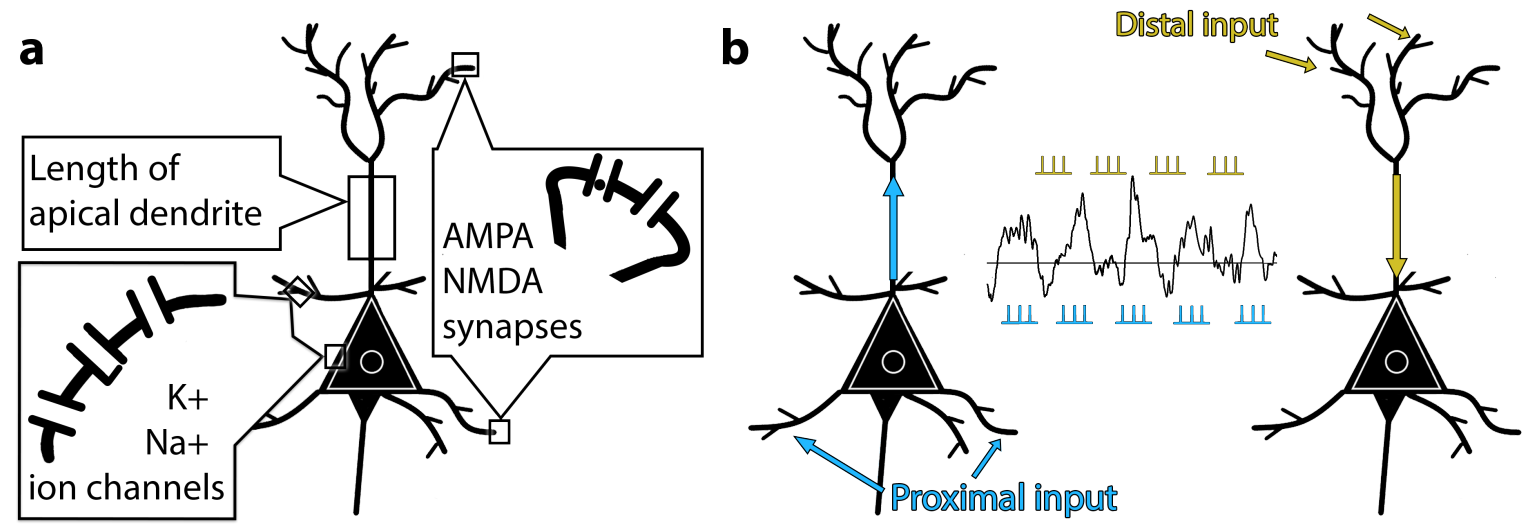

Figure 1: Schematic representation of a pyramidal neuron as it was modelled in Human Neocortical Neurosolver (HNN; Neymotin et al., 2020). a. Parameters of the model that were subjected to change: strength of incoming inputs via synaptic weights on AMPA and NMDA synaptic receptors, the density of voltage-gated sodium and potassium ion channels, and the length of apical dendrites. b. A schematic illustration of the effect that incoming inputs exert on the dendritic currents. The proximal drive is a simulation of thalamic activity coming to the granular layer of the cortex, which is later relayed to layer II/III and layer V. Distal drive is excitatory input from non-lemniscal thalamic sources and/or other cortical areas (see Methods). Proximal connections terminate on the basal dendrites of pyramidal neurons, while distal input is reaching apical dendrites. Therefore, currents that are generated in the neuron as a response to proximal stimulation flow predominantly in the upward direction (with respect to the surface of the cortex), and distal drive creates downward currents (Neymotin et al., 2020 ).

Further on, we simulated oscillations with both proximal and distal input and changed only one parameter at a time while holding others to default values (including random seed). We looked into the connection between biophysics and morphology of a pyramidal neuron in relation to the change of OM. Several important currents are modelled in HNN, but we took a closer look at a fast sodium current and a delayed rectifier potassium current, as primary currents responsible for depolarization and subsequent repolarization (Douglas and Martin, 

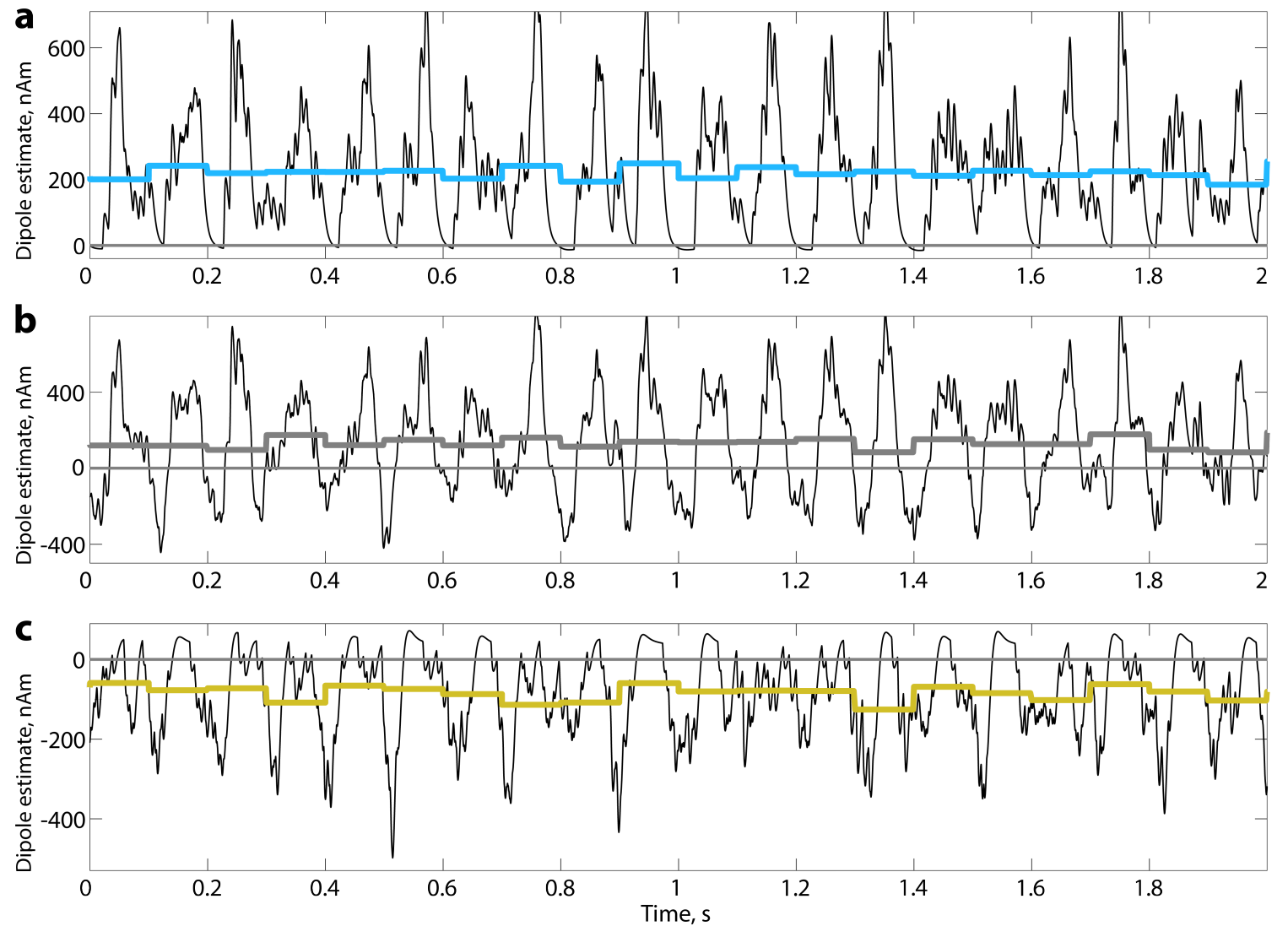

Figure 2: The alpha rhythm is generated in HNN with alternating rhythmic inputs (proximal and distal). a. Simulation of alpha rhythm with an isolated proximal input. b. Simulation of alpha rhythm with both proximal and distal input using default settings of the HNN model for the generation of the alpha rhythm. c. Simulation of alpha rhythm with an isolated distal input. Coloured line - OM that was computed on $100 \mathrm{~ms}$ intervals.

2004). Intuitively, when the density of sodium channels on the soma increases, they produce more inward current at the soma level. If all other channel densities are held constant, the increased current would flow along the apical dendrite, creating a more positive OM. On the opposite, an increase of the sodium current in dendrites would cause intracellular currents to flow down the apical dendrite and to the soma, thus forcing OM to shift towards less positive values. Figure $3 \mathrm{a}$ indeed confirms the assumption. While the model was generating alpha rhythm with interchanging proximal and distal inputs, an increase in sodium channel densities on the soma led to an increase in backpropagating currents and, subsequently, to an increase in OM. The tendencies for sodium and potassium channels had the opposite directions because sodium ions are moving inside the cell and potassium ions are leaving the cell.

Since the currents in the apical dendrite contribute primarily to the current dipole change (Neymotin et al., 2020), we hypothesised that extending the dendrite would result in a shift of OM. Extending the apical dendrite of pyramidal neurons in layer V indeed caused OM to drop below zero (Figure 3b). Layer V pyramidal neurons are larger than pyramidal neurons in layer II/III, and in particular, basal dendrites on the layer V pyramidal neurons are thicker (Bush and Sejnowski, 1993, Jones et al., 2007). Therefore, a current produced by proximal input 

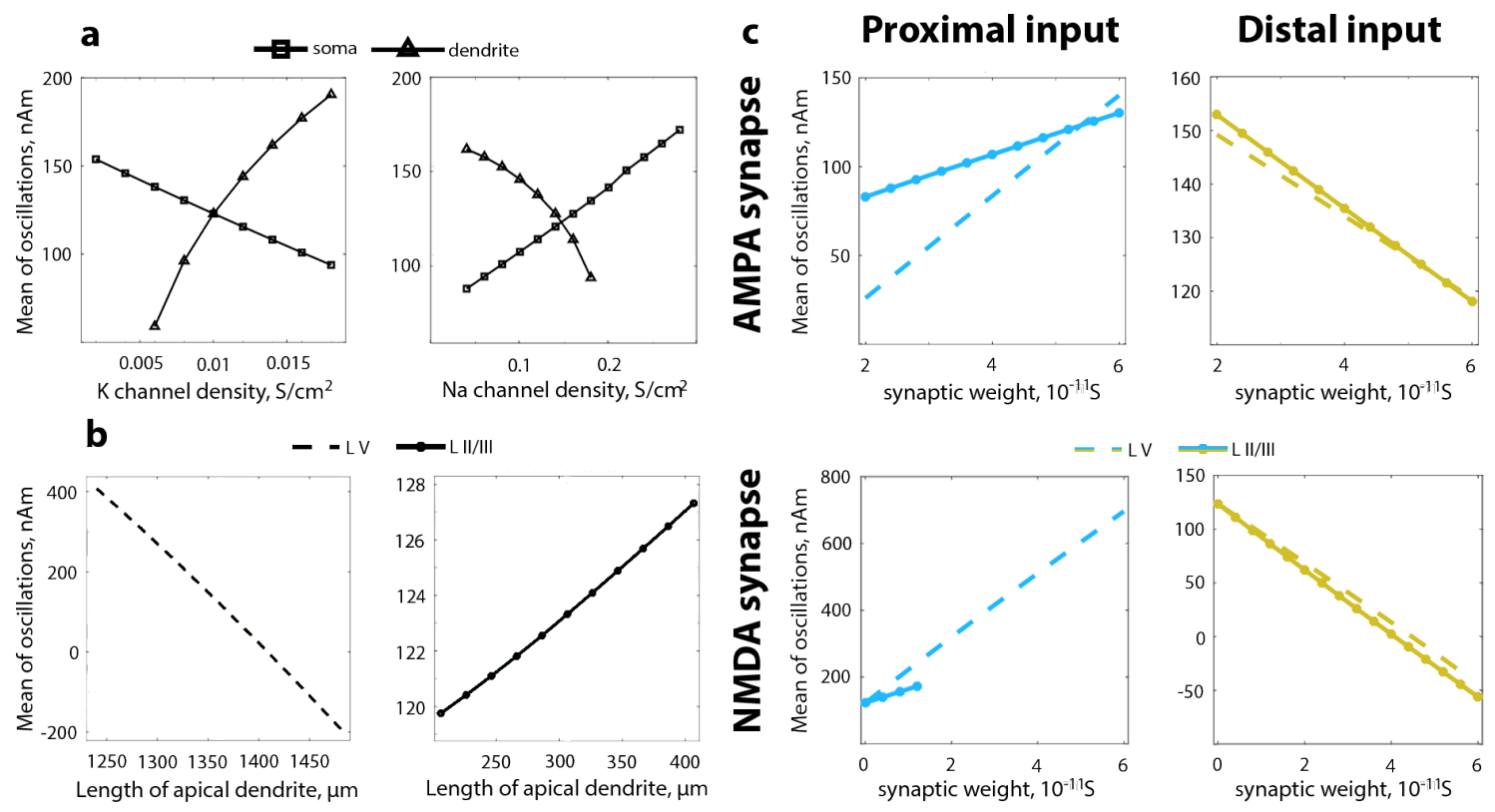

Figure 3: Relation between biophysics and morphology of a pyramidal neuron and OM. a. Change in $\mathrm{OM}$ of the alpha rhythm with a change in sodium and potassium channel density on soma and dendrites on pyramidal neurons in layer V. b. Change in OM with a change of the length of apical dendrite in pyramidal neurons in layer II/III and layer V. Note the y-axis range difference. c. Change in OM depending on the strength of incoming inputs realized through excitatory AMPA and NMDA synapses. Only one parameter at a time was changing, all other parameters were kept constant and set by default. OM is computed from 1-second of a simulated signal. L V - layer V, L II/III - layer II/III. The exact default values of parameters that were changed are in Methods, Table 2.

flows with less resistance through basal dendrites of pyramidal neurons in layer $\mathrm{V}$. When the length of the apical dendrite of pyramidal neurons in layer $\mathrm{V}$ is increased this upward current encounters more resistance in the dendrite and thus undergoes a decrease in its strength. For pyramidal neurons in layer II/III, this is not the case since their upward current produced by proximal input is already constrained with thin basal dendrites and an increase in the length of apical dendrite of pyramidal neurons in layer II/III and a subsequent very small increase in OM (Figure 3b) reflect impediments for the current originated from distal input. With these simulations, we demonstrate what has been predicted by previous research (Nikulin et al., 2007. Mazaheri and Jensen, 2008) that currents in pyramidal neurons are asymmetric and, consequently, elongating apical dendrite produced even more asymmetry.

Lastly, we varied weights of the AMPA and NMDA synaptic receptors for both proximal and distal inputs (Figure 33; the ranges for changes are similar as in Jones et al., 2009). The high synaptic weight corresponds to more stimulation coming from a particular input which in turn should be associated with an increase in currents. The proximal drive arrives to basal dendrites of a pyramidal neuron, and currents that are generated in the neuron as a result of proximal stimulation flow upwards (in relation to the cortical surface). Therefore, a bigger stimulation from the proximal sources induced an increase in OM (Figure 3., left column). The distal drive gives rise to downward currents, and an increase in the distal drive 
is associated with a decrease in OM (Figure 3c, right column). The tendency was matching for both AMPA and NMDA. AMPA receptors have fast time constants, while NMDA receptors have long time constants (Buzsáki et al., 2012), therefore, a change in the synaptic weight of NMDA receptors gave rise to a larger change in $\mathrm{OM}$ due to a larger effect on the currents. Note that for some cases increase in weights of NMDA receptors causes spiking in the network. For that reason, further change in $\mathrm{OM}$ is not presented in Figure 3 . (lower left panel).

To simulate the amplitude of a signal that is typically observed in microscopic recordings, oscillations of 200 pyramidal neurons are then multiplied by 300,000 (Neymotin et al., 2020) so that $60,000,000$ pyramidal neurons give rise to the signal. However, in real settings, the number of synchronously oscillating neurons is rarely constant. Instead, it fluctuates over time (Palva and Palva, 2007). This decrease or increase on the microscopic level is reflected in the changes of the oscillations' amplitude (Elul, 1972, Pfurtscheller and Da Silva, 1999). Having that in mind, we extracted the amplitude envelope of the alpha rhythm from real exemplary EEG data and modulated the number of neurons in the model with the extracted envelope. Afterwards, we estimated baseline-shift index (BSI; Nikulin et al., 2010; see Methods), a measure that allows to detect baseline shifts associated with a non-zero OM in empirical electrophysiological data. As predicted, because OM was positive, the BSI turned out to be positive as well (Figure $4 \mathrm{a}, \mathrm{d}$ ). For this simple simulated case, we demonstrate that there exists a straightforward dependence between the amplitude of alpha rhythm and low-frequency amplitude (Figure 4 4 ) that can be measured with BSI.

a
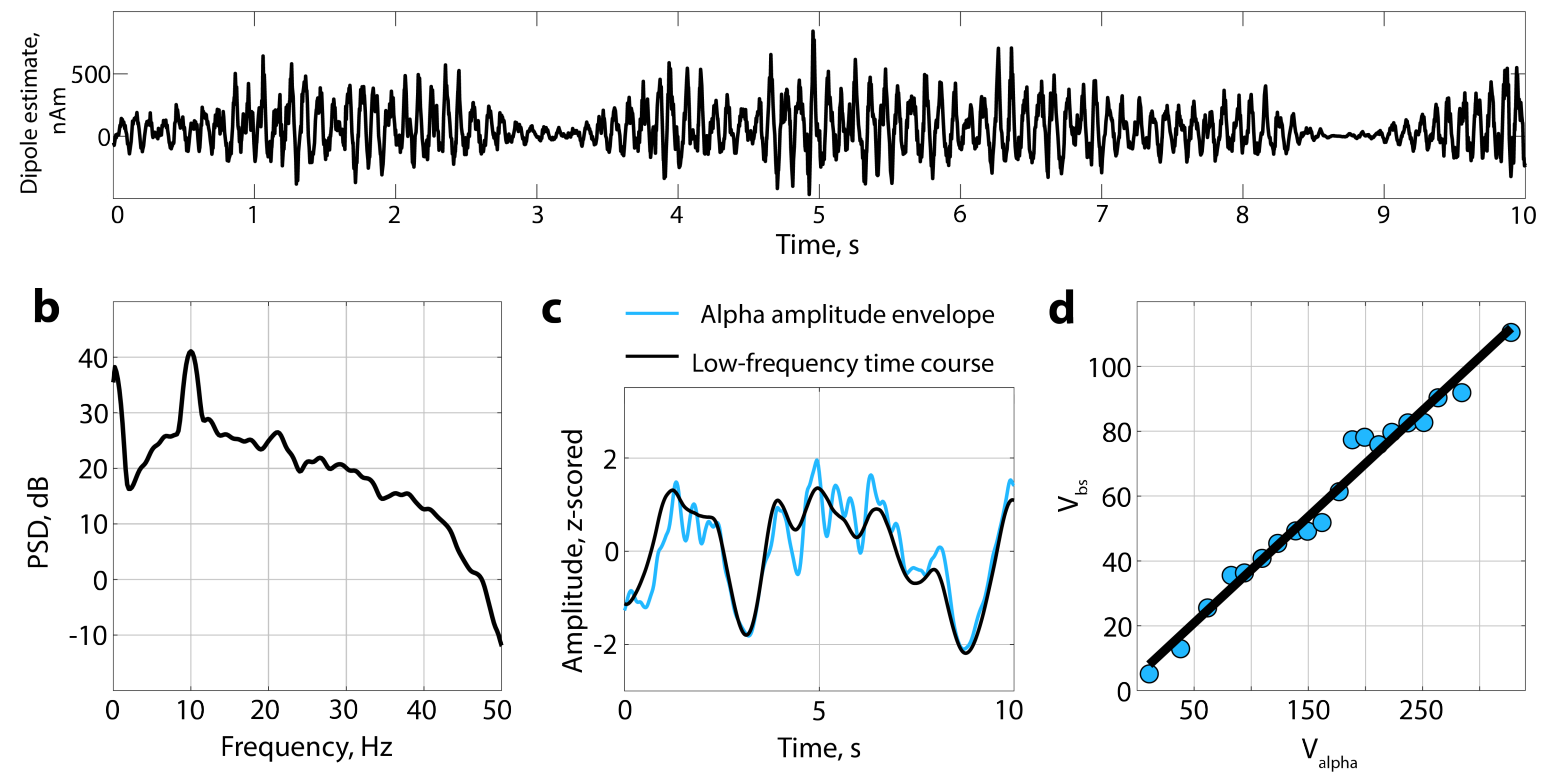

Figure 4: Alpha modulation from real EEG is superimposed on time series simulated with default settings in HNN. a. Modulated oscillations. b. A spectrum of the modulated signal. c. Correspondence between alpha amplitude envelope and simulated time course filtered in a low-frequency range. Since $\mathrm{OM}$ is positive, an increase in alpha amplitude is associated with a baseline shift upwards. d. Baseline-shift index (BSI; Nikulin et al., 2010) applied to a simulated and modulated signal. The sign of BSI is positive which is consistent with $\mathrm{OM}$ of simulated oscillation. BSI computed as a Pearson coefficient is 0.98 . 


\section{Validating the baseline-shift mechanism on the large EEG data set}

To further validate the presence of non-zero mean in neuronal oscillations we analyzed data from 90 participants (50 young and 40 elderly participants). From the broadband EEG data, we extracted oscillatory components with Spatio-Spectral Decomposition (SSD; Nikulin et al., 2011), and chose the first five SSD components that typically have the strongest signalto-noise ratio in the alpha band. The total number of time courses extracted was 900 (500 for young and 400 for elderly participants in two conditions - eyes-closed and eyes-open). Figure 5 shows the representative example of three participants: spatial distribution of the SSD component (Figure 5a), its spectrum (Figure 5b), a baseline-shift index (BSI; Figure 5;), and a corresponding baseline shifts in low frequency associated with the modulation of the alpha rhythm (Figure 5d). For these examples, the BSI or the correlation between alpha amplitude envelope and low-frequency amplitude is more than 0.9 (BSI was computed as the Pearson correlation coefficient). In general, more than $30 \%$ of obtained BSIs (39\% for young participants, $31 \%$ for elderly participants) demonstrated a strong correlation $(|\mathrm{BSI}|>$ 0.7 ), and around 30\% (31\% for young participants, $30 \%$ for elderly participants) - moderate correlation $(0.4<|\mathrm{BSI}|<0.7$; the interpretation of strength of the correlation is based on Dancey and Reidy, 2007).

Absolute BSI values had a tendency to be larger in the young group $(0.564 \pm 0.012$ in the young group, $0.494 \pm 0.015$ in the elderly group when averaged across conditions \pm standard error of the mean). Figure 6 shows the corresponding data. To test whether the difference in absolute BSIs was significant between the groups and conditions we applied ANOVA. Additionally, we extended the ANOVA model with covariate variables such as power ratio in the alpha band and the power ratio in the low-frequency band that are known to differ in age groups and might have an effect on BSI. In our sample, power ratio in the alpha band was significantly different between the age groups and conditions (ANOVA for age groups $F=16.62, p<0.001, \eta^{2}=0.015$; conditions $F=194.78, p<0.001, \eta^{2}=0.175$; no significant interaction $F=3.69, p=0.06, \eta^{2}=0.003$ ), as well as power ratio in the low-frequency band (ANOVA for age groups $F=10.61, p<0.002, \eta^{2}=0.011$; conditions $F=19.41, p<0.001$, $\eta^{2}=0.021$; no significant interaction $\left.F=0.03, p=0.86, \eta^{2}=0.000\right)$. On average, the power ratio in the alpha band was higher in the young group and in the eyes-closed condition. Conversely, the power ratio in the low-frequency band was higher in the elderly group and in the eyes-open condition. Based on ANOVA with absolute BSI as a dependent variable, the difference between age groups was significant $\left(F=10.50, p<0.002, \eta^{2}=0.011\right.$; post hoc Wilcoxon test for age groups: $p<0.001)$. The difference between eyes-open and eyes-closed conditions failed to reach a significance threshold $\left(F=2.64, p=0.10, \eta^{2}=0.003\right)$, as well as no interaction in age-condition pairs was detected $\left(F=3.17, p=0.08, \eta^{2}=0.003\right)$. In addition, in ANOVA, the power ratio in the alpha band and power ratio in the low-frequency band were not strongly associated with the dependent variable (power ratio in the alpha band: $F=2.28, p=0.13, \eta^{2}=0.002$; power ratio in the low-frequency band: $F=4.68, p=0.03$, $\eta^{2}=0.005$; correlation values between covariates and BSI in age groups and conditions are presented in Table 1). We also run ANOVA on the mean values of BSI (averaged within subject-condition) and obtained similar results - absolute values of BSI were significantly different between age groups $\left(F=9.29, p<0.003, \eta^{2}=0.049\right)$. 

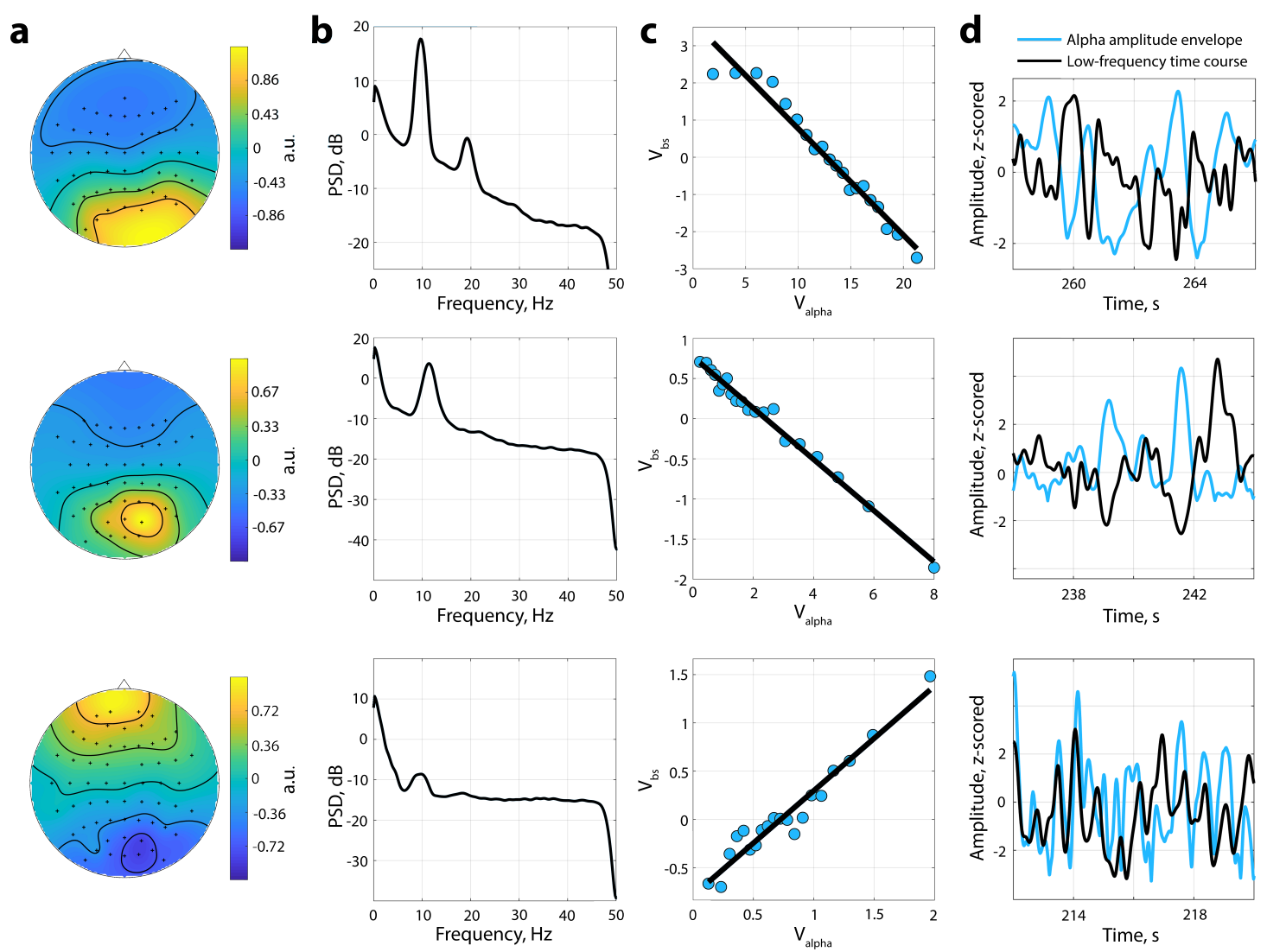

Figure 5: Baseline shifts in rest EEG data. Examples of obtained SSD components topographies (Column a) and spectrums (Column b). Column c. The association between alpha amplitude envelope $\left(V_{a l p h a}\right)$ and low-frequency amplitude $\left(V_{b s}\right)$ - baseline-shift index (BSI). BSI can be estimated as a slope of linear regression or as a correlation coefficient. For exemplar components of the first two participants, the BSI is negative, for the last one - positive. Column d. Correspondence between alpha amplitude envelope and low-frequency signal. If BSI $<0$, an increase in the alpha amplitude produces a baseline shift downwards. Contrary, if BSI $>0$, an increase in the alpha amplitude produces a baseline shift upwards.

Table 1: Correlation between the absolute value of BSI versus power ratio in the alpha band or power ratio in the low-frequency band. The correlation was computed with the Pearson correlation coefficient. The p-value is presented in the brackets.

Young group Elderly group

\begin{tabular}{lcccc} 
& eyes-closed & eyes-open & eyes-closed & eyes-open \\
\hline power ratio in the alpha band & $\rho=0.16$ & $\rho=0.14$ & $\rho=-0.07$ & $\rho=0.02$ \\
& $(p<0.013)$ & $(p<0.03)$ & $(p=0.36)$ & $(p=0.81)$ \\
\hline power ratio in the low-frequency band & $\rho=-0.09$ & $\rho=-0.11$ & $\rho=0.00$ & $\rho=-0.17$ \\
& $(p=0.15)$ & $(p=0.08)$ & $(p=0.95)$ & $(p<0.02)$
\end{tabular}

\section{Effects of spatial synchronization on the degree of alpha amplitude modu- lation and baseline shifts}

Our EEG findings indicate that the power ratio only weakly correlates with the magnitude of BSI. It means that there can be neuronal factors affecting differently the amplitude 


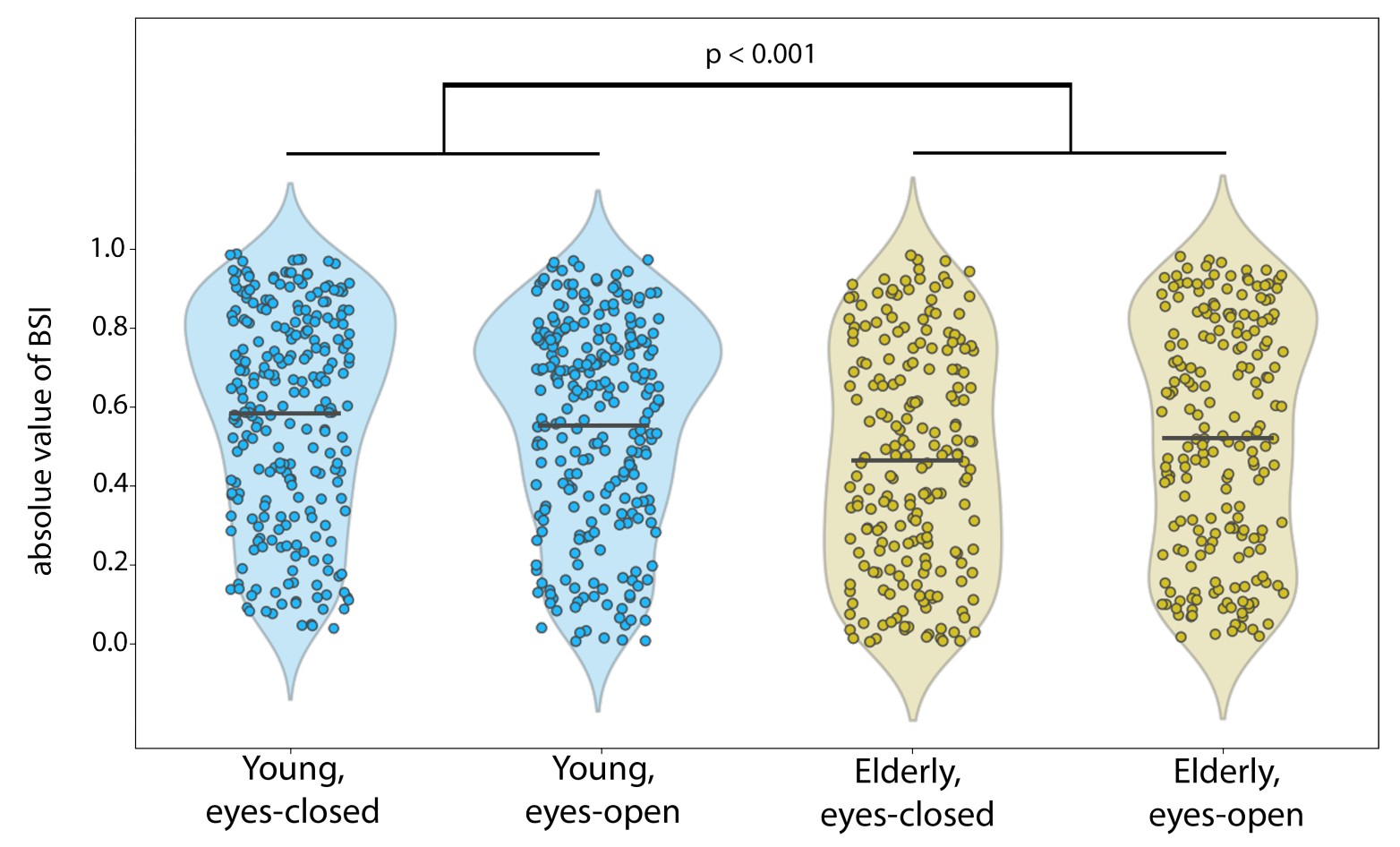

Figure 6: A difference in the means of absolute values of BSI. BSI was computed as the Pearson correlation and compared between different groups/conditions. Each dot represents an absolute BSI of one SSD component of each participant. BSI was significantly larger for young participants compared to elderly participants. $p<0.001$ is based on post hoc Wilcoxon test for age groups.

of oscillations and baseline shift. One possible factor is spatial synchronization among the neurons generating alpha oscillations (Schaworonkow and Nikulin, 2019). Using population modelling, we show that the degree of spatial synchronization has indeed a strong impact on the amplitude of macroscopic alpha oscillations while not having a considerable influence on the generation of the baseline shifts.

For the case of a synchronous network, both alpha modulation and evoked response were present after averaging over epochs (Figure 7, left column). A clear alpha modulation is visible even on a single epoch level. Note that each epoch started with a new phase distribution of individual oscillators, and the central value for distribution was selected randomly (Figure 7, upper left panel, the central value for the displayed epoch is marked by black arrow). Therefore, alpha oscillations in the evoked response time course (Figure 7, last panel, after averaging of all epochs) were almost cancelled out. Yet baseline shift, representing evoked response, remained since it is not sensitive to the phase of individual oscillators.

For the case of an asynchronous network, alpha modulation was no longer visible even for a single epoch, but the evoked response remained prominent (Figure 7, right column). Visible signs of the modulation of the alpha rhythm (modulation of the amplitude envelope) had mostly disappeared in the time course of one epoch, and even more so in the amplitude envelope averaged over 100 epochs. The attenuation of macroscopic oscillations was due to the phase cancellation among individual oscillators. On the contrary, as simulated oscillators had a non-zero OM of the same magnitude as in the previous case (for the synchronous network), the evoked-response time course replicated the one from the synchronous network simulation, 
except for the residual alpha oscillations. This is due to the fact that the amplitude of neuronal oscillations critically depends on the phase synchronization while baseline shifts require only the amplitude modulation of non-zero mean oscillations which is not sensitive to the phase of ongoing oscillations.

\section{Synchronous network}
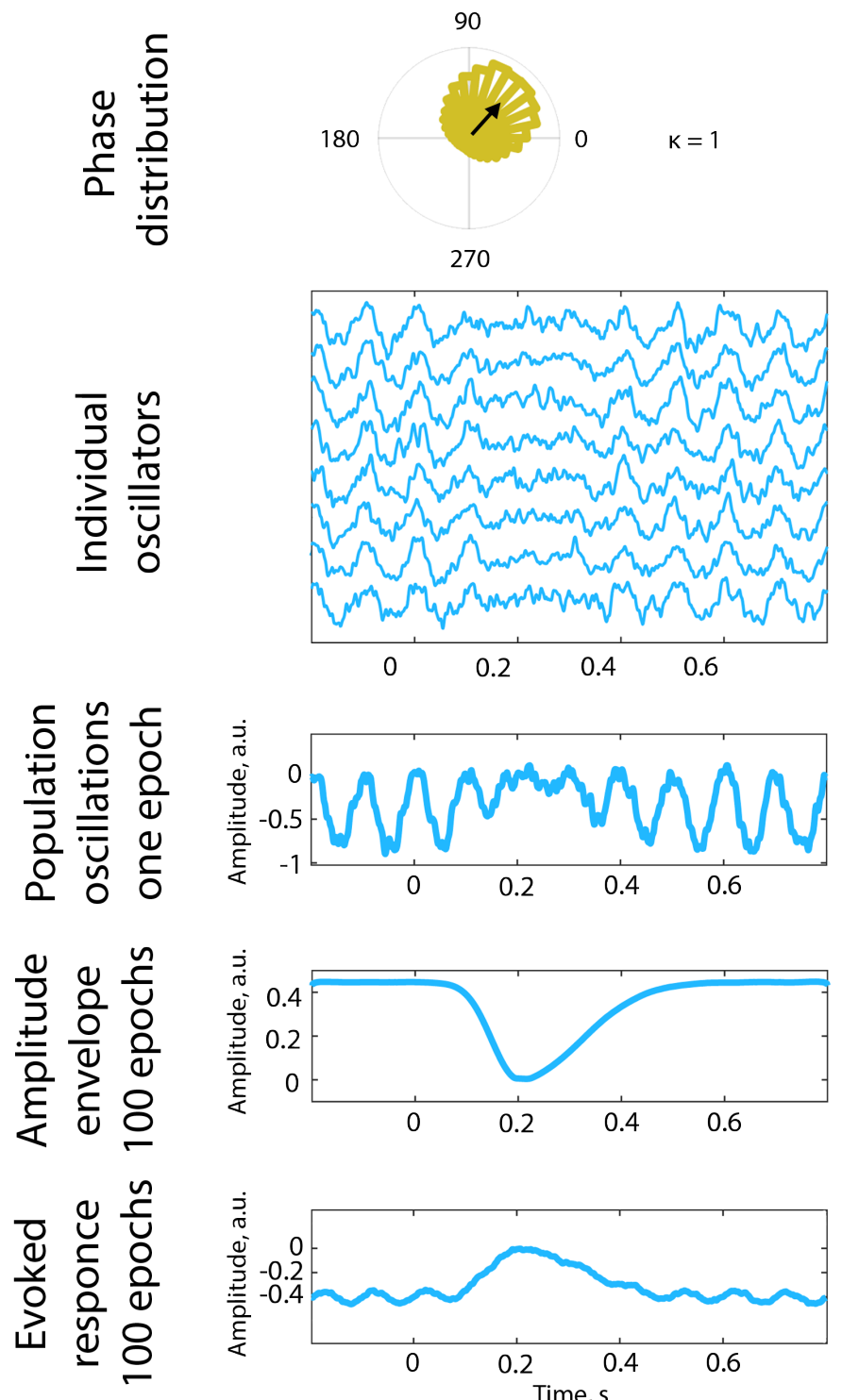

\section{Asynchronous network}
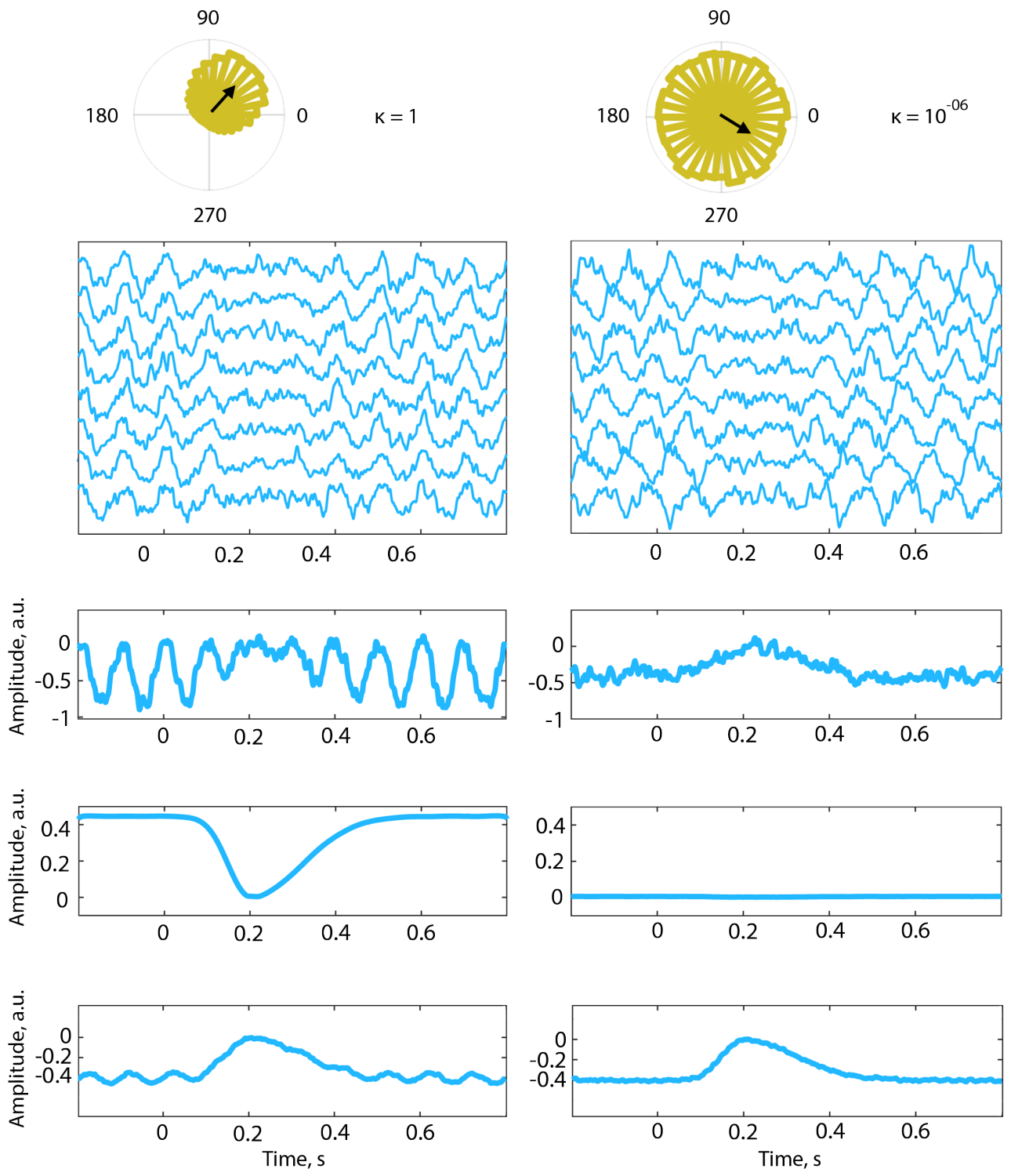

Figure 7: Synchronous and asynchronous networks. For phase distribution of the individual oscillators at the beginning of a simulation, phases were sampled from von Mises distribution. The central value of the distribution (or preferred direction) for the displayed epoch is marked by the black arrow. $\kappa$ is the concentration parameter that tunes the spread of phases at the beginning of an epoch. Amplitude modulation was modelled as inverted Gaussian with varying widths of the left and right planks. The network contained 30000 oscillators. 


\section{Discussion}

A non-zero oscillatory mean (OM) can be conceptualized on a neuronal level as an average value of fluctuations in electrical or magnetic fields produced by neuronal currents. When the ongoing rhythm with underlying non-zero OM is modulated, the baseline shift appears in the frequency range corresponding to a frequency of the amplitude modulation. Therefore, the presence of baseline shifts in real electrophysiological data allows inferring the presence of a non-zero OM. This concept, if valid, has numerous implications for data analysis of EEG/MEG signals and for the interpretation of diverse experimental results.

In the current research, we obtained supporting evidence for the hypothesis of a nonzero OM with the use of computational models and analysis of the real EEG recordings. The Human Neocortical Neurosolver (HNN; Neymotin et al., 2020), the software and the underlying model of a cortical patch was previously validated for human neurophysiological data, including the investigation of mu-rhythm (Jones et al., 2009). In our simulations with HNN, we show that pyramidal neurons in layer II/III and layer V of the neocortex generate a current dipole that has a non-zero OM when being integrated over a few oscillations' periods. The non-zero OM was present for diverse settings relating to synaptic conductances, voltagegated channel densities, and the length of apical dendrites, and the OM variations were consistent with the changes in the distribution of currents inside the neuron. There are other parameters in the model that affect neuronal functioning that we did not discuss. For instance, we did not present results for other channel densities such as channels for $\mathrm{Ca}^{2+}$ because their contribution to the generation of a current dipole was small. In fact, the corresponding changes in $\mathrm{OM}$ were several orders of magnitude smaller for $\mathrm{Ca}^{2+}$ compared to $\mathrm{K}^{+}$and $\mathrm{Na}^{+}$currents. Besides, as proximal and distal inputs were driven exclusively by excitatory spiking, we did not present results of other neurotransmitters such as GABA, acetylcholine, noradrenaline etc. In general, for this demonstration, we selected the parameters that, in our view, have the strongest contribution to the primary currents relating to postsynaptic potentials responsible for the generation of alpha oscillations. For almost all settings of tested parameters, OM was always non-zero, thus indicating that zero OM should rather be an exception and typically alpha oscillations should primarily be associated with non-zero OM.

In these simulations, we consider the optimal conditions where the simulated population of neurons is oriented in the same direction, which in turn creates an ideal situation for the detection of asymmetric currents. In the case of a real brain, primary currents of different directions are present due to the intricate folding of the cortex. And such mixing of sources may lead to OM closer to zero. To further validate the presence of non-zero OM, we also analyzed a large data set containing resting-state EEG recordings.

There, we estimated a non-zero OM with a baseline-shift index (BSI; Nikulin et al., 2010), which in this study was computed as the Pearson correlation coefficient. More than $30 \%$ of obtained BSIs demonstrated a strong correlation $(|\mathrm{BSI}|>0.7)$, and around $30 \%$ - moderate correlation $(0.4<|\mathrm{BSI}|<0.7)$, thus in general suggesting the ubiquity of the non-zero OM phenomenon. When comparing differences in average values of absolute BSIs in age groups and conditions we found that elderly participants had smaller BSIs. This could have been explained by the fact that alpha power decreases with age (Rossini et al., 2007, Schomer and Da Silva, 2010), which was also the case for our sample, and high power may lead to a more robust estimation of BSI due to higher signal-to-noise ratio. We controlled for power in the ANOVA model (with power ratio in the alpha band), but significant difference between age groups remained. Possibly for the elderly group, spatial filtering in SSD resulted in extracted components that represented a larger mixture of alpha sources with varying in sign 
baseline shifts compared to younger participants. Additionally, the power in low frequency was higher in the elderly group (the finding that some researchers associate with both healthy and pathological aging; Ishii et al., 2017). Since BSI takes into consideration both alpha oscillations and the low-frequency signal, the noise in the low-frequency range may affect the estimation of BSI thus making the detection of an association between alpha oscillations and baseline shifts difficult for elderly participants.

In our analysis of real EEG, we observed that the correlation between the relative power of alpha and BSI magnitude was not strong. This indicates that there should also be other factors that can explain a discrepancy between the fluctuation of amplitude envelopes and corresponding baseline shifts. We hypothesized that one of such influential factors can be the strength of spatial synchronization.

Therefore, we tested such spatial synchronization assumption in a population model of simple oscillators simulated as sinusoids with a non-zero offset. In the case of a strong spatial synchronization, alpha oscillations with non-zero OM demonstrate both pronounced amplitude modulation and corresponding baseline-shift dynamics. On the contrary, reduced spatial synchronization provided a case when a low-frequency baseline shift was still present, but no amplitude modulation was detected. Subdural recordings research in humans (Bullock et al., 1995) and animal studies (Destexhe et al., 1999) demonstrated a rapid decrease in the coherence between neuronal columns with a distance of just a few millimetres. This in turn indicates that oscillatory sources can be active simultaneously but without considerable spatial phase synchronization thus leading to the attenuation of macroscopic oscillatory amplitude because of the phase cancellations. Such attenuation of amplitude makes it also hard to detect amplitude modulation. Yet baseline shifts are insensitive to the phase alignment of the individual oscillatory sources and therefore the change in the temporal dynamics of the baseline shifts are unaffected by the spatial synchronization. As a consequence, the prediction of a baseline-shift mechanism of evoked response generation (BSM) that alpha modulation always accompanies evoked response may not be easily observed in empirical EEG/MEG recordings. Importantly, in our simulations, even for the low spatial synchronization, amplitude modulation was noticeable in the time course of one oscillator. This kind of data is unavailable with the extracranial recordings. Intracranial single- or multi-unit methods may provide a missing link between neuronal level and macroscopic observations, especially for the case of the asynchronous networks.

These simulations demonstrated that in addition to a case when both evoked response and alpha amplitude modulation are observed, it is also possible to observe evoked response without the detectable amplitude modulation of oscillations (although it is present at the level of individual sources). However, it is an unlikely scenario that the amplitude modulation is present yet no evoked response is detected (apart from the trivial case when neuronal oscillations have a zero OM).

BSM provides far-reaching implications for other phenomena that are investigated in neuroscience. For instance, to a certain degree, phase-amplitude coupling might be a reflection of the modulation of high-frequency activity (with non-zero OM) in a low-frequency range. In this scenario, if the amplitude of alpha oscillations is modulated in a delta frequency range, the phase of baseline shifts in the delta rhythm would be coupled to the increases/decreases in the amplitude of alpha oscillations. Critically, it means that low-frequency oscillations carry relevant information about a high-frequency activity (here we use high-frequency and lowfrequency as relative terms, not predetermined ranges), and they both should not be treated as isolated events. For instance, several studies pointed to the role of ultra-slow oscillations in sensory processing (Monto et al., 2008, Demanuele et al., 2013), but the association be- 
tween ultra-slow oscillations and alpha rhythm was not explored. However, possibly, those correlations partially stemmed from a non-zero mean theta or alpha oscillations which, when modulated, induced baseline shifts in the slow activity. Importantly, this effect may relate to other frequency bands. Alpha-gamma phase-amplitude coupling is one of the incidences of cross-frequency coupling (Osipova et al., 2008) that may also partially result from the presence of gamma baseline shifts.

Although so far, we were discussing only alpha oscillation, it is plausible that beta- and gamma-band activity also fluctuates around a non-zero OM. There is evidence of high beta and gamma that coincide with early visual evoked responses (Tzelepi et al., 2000), and even more so, the modulation of a non-zero mean gamma can potentially underlie early evoked responses generation (Mazaheri and Jensen, 2008).

Many previous studies provided evidence that late evoked response components, for instance, P300, contingent negative variation (CNV), N400, earlier left anterior negativity (ELAN) and others, occur concurrently with the changes in alpha rhythm amplitude (Bender et al., 2004, Ergenoglu et al., 2004, Hald et al., 2006, Hong et al., 2015, Heimann et al., 2017, Amit et al., 2019). Additionally, movement related responses such as readiness potential, also coincide with a decrease in the power of the alpha rhythm (Shibasaki and Hallett, 2006). Other research teams assessed cognitive functioning with the simultaneous evoked response and event-related desynchronization (ERD), with the results showing consistent correlation of evoked response amplitude and/or latency with the corresponding parameters of ERD in the alpha band (Yordanova et al., 2001, Funderud et al., 2012, Abeles and Gomez-Ramirez, 2014, Störmer et al., 2016. Schneider and Maguire, 2018). All these findings can be to a certain extent explained on the basis of the results of our study.

Importantly, BSM can coexist with the additive mechanism where evoked responses are generated by the activation of neurons without the concurrent changes in oscillatory dynamics, and with the phase-reset mechanism where after stimulus presentation phases of oscillations are shifted and become aligned. However, BSM may explain not only the evoked response per se but also the change in amplitude of evoked response, when it is generated due to a combination of mechanisms.

In the current study, we observed that amplitude modulation of the alpha oscillations is reflected in the low-frequency component as predicted by BSM, which is the evidence for the existence of a non-zero OM in alpha oscillations. This in turn provides further support for the idea that evoked responses and neuronal oscillations share common neurophysiological mechanisms. Our results thus indicate that a number of motor and cognitive responses which coincide in time with the modulation of alpha oscillations may be interpreted in the context of oscillatory neuronal dynamics.

\section{Methods}

The main prerequisites for BSM are 1) the non-zero OM and 2) amplitude modulation of oscillations (Nikulin et al., 2007). This mechanism can be summarized with the equation:

$$
\begin{gathered}
x=\sum_{i=1}^{M} S_{i} \\
S_{i}=A(t)[\cos (2 \pi f t+\theta)+r]=A(t) \cos (2 \pi f t+\theta)+A(t) r
\end{gathered}
$$

where $S_{i}$ - data from a single oscillator s, 
$f$ - some arbitrary frequency of oscillations in the population,

$\theta$ - some arbitrary phase,

$r$ - non-zero OM,

$A(t)$ - amplitude modulation,

$A(t) r$ - baseline shift that accompanies oscillations,

$M$ - number of oscillators.

When averaging is performed, phases of oscillations across trials cancel each other, but the baseline shifts persist and emerge on a summed signal. The shift may have a positive or negative direction depending on the orientation of a dipole and on the sign of an underlying OM (Nikulin et al., 2007, Mazaheri and Jensen, 2008, Iemi et al., 2019).

In the current study, we adopted an integral strategy and attempted to demonstrate the following: 1) asymmetric currents lead to a non-zero $\mathrm{OM}$ in alpha oscillations ( $r$ term in eq. 1 is not equal to zero); 2) if oscillations possess the property of a non-zero OM, modulation affects $\mathrm{OM}$ as well, thus leading to the emergence of a baseline shift in lower frequency $(A(t)$ term is present both in amplitude envelope and in low-frequency component); 3) the prominence of amplitude modulation may change due to spatial synchronization and, therefore, affect the relationship between amplitude modulation and corresponding baselineshifts $(A(t)$ in $A(t) \cos (2 f t+\theta)$ may not be evident in EEG/MEG data).

\section{Simulation of the alpha rhythm with a biophysical model}

In this study, a biologically plausible model of neuronal network generating alpha rhythm was selected to explore a non-zero property of oscillations. Human Neocortical Neurosolver (HNN), a software tool and an underlying model was developed by Stephanie R. Jones laboratory in Brown University (Neymotin et al., 2020) for investigation of cellular mechanisms of both ongoing and evoked activity. Here we provide a brief description of neuronal architecture and principles behind the generation of alpha rhythm as it was built in HNN. For a more detailed report, please refer to Jones et al., 2000, Jones et al., 2007, Jones et al., 2009, Lee and Jones, 2013, Neymotin et al., 2020.

The model consists of pyramidal neurons and interneurons in layer II/III and layer V of the neocortex, with the ratio of pyramidal neurons to interneurons as three to one and a total count of 270 cells. Each pyramidal cell is modelled as having a soma and a dendritic tree that consists of several compartments (Jones et al., 2009). Interneurons are known to contribute significantly less to the potential/ field; hence they are modelled as a single somatic compartment cell. For each compartment, membrane voltage is computed from the standard Hodgkin-Huxley equations, and current between compartments flows according to cable theory (Neymotin et al., 2020). Biophysically realistic flow of ions inside the neuron is achieved by simulating several active currents: a fast sodium current, a delayed rectifier potassium current, an adapting potassium current, and a leak current in pyramidal neurons in layer II/III, same currents and a calcium current, a potassium-activated calcium current, a low-threshold calcium current and a hyperpolarization-activated mixed cation current in pyramidal neurons in layer V (Jones et al., 2007, Jones et al., 2009). The strength of currents can be adjusted in the interface by setting the channel density for each current. In addition to active currents, each cell receives excitatory and inhibitory input from the neighbours. Local dynamics in the network is realized via AMPA, NMDA and GABAa,b synaptic receptors.

Several exogenous drives are available for investigation - evoked inputs, rhythmic inputs, Poisson and tonic drives. Rhythmic inputs are of the most interest for us because they initiate 
ongoing oscillations. Rhythmic drive is simulated as spike trains coming from "outside", it is excitatory and realized through AMPA and NMDA synaptic receptors (Neymotin et al., 2020). These rhythmic inputs come from two directions - proximal and distal. Proximal drive represents thalamic stimulation, and it arrives at both layer II/III and layer V pyramidal neurons, with the latter connection being delayed (0.1 ms to layer II/III and $1.0 \mathrm{~ms}$ to layer V). Proximal input connections contact basal dendrites of pyramidal cells and hence generate current flowing in the apical dendrite in an upward direction. The distal drive is a stimulation coming from other cortical areas and non-lemniscal thalamic sources. It reaches apical dendrites of pyramidal neurons in both cortical layers simultaneously and induces current flowing down the apical dendrite (Jones et al., 2009). The strength of inputs is controlled with the weights of the AMPA and NMDA receptors, and the number of bursts in the spike train. Spontaneous alpha rhythm emerges when the delay between proximal and distal input is exactly $50 \mathrm{~ms}$, and they are delivered to the network with a frequency of $10 \mathrm{~Hz}$ (Ziegler et al., 2010, Kerr et al., 2013, Neymotin et al., 2020). The main output of the simulation is a current dipole estimate. The current dipole is computed as the sum of the intracellular currents within the dendrites projected onto the direction parallel to the apical dendrite plane. The current dipole quantifies units of current per unit of distance, therefore its measurement unit is Am (Ampere x meter; Neymotin et al., 2020).

To investigate the effect of currents on OM, we varied several parameters in the model (Figure 1), such as synaptic conductances, voltage-gated channel densities, length of dendrites, and intensity of incoming stimuli (through a change in the synaptic weights). The default values of parameters that were subjected to change are presented in Table2, We hypothesised that if unbalanced currents lead to oscillations with a non-zero OM, the change in currents will correspond to a change in OM of a generated signal.

Additionally, to test BSM assumption, we modulated a simulated time course by means of multiplication with an amplitude envelope extracted from real EEG data: $X(t)=A(t) m(f, t)$ where $A(t)$ - the alpha amplitude envelope extracted from EEG data with the Hilbert transform, $m(f, t)$ - oscillations that were produced by the model with $f=10 \mathrm{~Hz}$, and $X(t)$ modulated signal. Afterwards, using the modulated oscillations we computed the baselineshift index. The method for estimating the baseline-shift index is presented in the next subsection.

\section{Estimation of baseline shifts in EEG data}

\section{Participants}

Analysis of empirical data was performed on Leipzig Study for Mind-Body-Emotion Interactions (LEMON; Babayan et al., 2019) data set, which contains EEG data from 216 healthy participants of young $(25.1 \pm 3.1$ years, range $20-35$ years $)$ and old age ( $67.6 \pm 4.7$ years, range 59-77 years). Resting-state EEG was recorded from 62 channels placed according to the 10-20 extended localization, referenced to $\mathrm{FCz}$ and grounded at the sternum. The EEG session was carried with alternating 60 -sec blocks of eyes-open and eyes-closed conditions, comprising a total of 16 segments starting from the eyes-closed state. In the current study, we used data from young, from 20 to 25 years, and elderly participants, from 60 to 80 years. The choice of participants from the data set was performed based on the following criteria - no habit of smoking, no drug administration at the time of sampling, no depression (based on the Hamilton Depression scale) or any other current mental condition. Additionally, 12 recordings were removed due to significant noise in several channels or the absence of alpha oscillations in the spectrum. After such exclusion, data from 50 young participants and 40 elderly participants 
Table 2: Default parameters of the HNN model (Neymotin et al., 2020). PN II/III- pyramidal neuron in layer II/III, PN V- pyramidal neuron in layer V

\begin{tabular}{|c|c|}
\hline Parameter & Value \\
\hline \multicolumn{2}{|l|}{ Morphology of the Pyramidal neuron } \\
\hline PN II/III - Apical dendrite length $(\mu m)$ & 306.0 \\
\hline PN V - First segment of apical dendrite length $(\mu m)$ & 680.0 \\
\hline PN V - Second segment of apical dendrite length $(\mu m)$ & 680.0 \\
\hline \multicolumn{2}{|l|}{ Biophysics of the Pyramidal neuron in layer V } \\
\hline Soma K+ channel density $\left(S / \mathrm{cm}^{2}\right)$ & 0.01 \\
\hline Soma $\mathrm{Na}+$ channel density $\left(\mathrm{S} / \mathrm{cm}^{2}\right)$ & 0.16 \\
\hline Dendrite $\mathrm{K}+$ channel density $\left(S / \mathrm{cm}^{2}\right)$ & 0.01 \\
\hline Dendrite $\mathrm{Na}+$ channel density $\left(\mathrm{S} / \mathrm{cm}^{2}\right)$ & 0.14 \\
\hline \multicolumn{2}{|l|}{ Rhythmic proximal inputs } \\
\hline PN II/III AMPA weight $(\mu S)$ & 0.000054 \\
\hline PN II/III NMDA weight $(\mu S)$ & 0.0 \\
\hline PN V AMPA weight $(\mu S)$ & 0.000054 \\
\hline PN V NMDA weight $(\mu S)$ & 0.0 \\
\hline \multicolumn{2}{|l|}{ Rhythmic distal inputs } \\
\hline PN II/III AMPA weight $(\mu S)$ & 0.000054 \\
\hline PN II/III NMDA weight $(\mu S)$ & 0.0 \\
\hline PN V AMPA weight $(\mu S)$ & 0.000054 \\
\hline PN V NMDA weight $(\mu S)$ & 0.0 \\
\hline
\end{tabular}

were separated into eyes-open and eyes-closed conditions and BSI estimation was performed as described in the next subsection.

\section{The baseline-shift index}

For the most part, we applied the method for inferring baseline shifts in empirical electrophysiological recordings as described by Nikulin et al., 2007, Nikulin et al., 2010 with some adjustments. The analysis was performed in MATLAB (R2018b, MathWorks) using the BBCI Toolbox (Blankertz et al., 2016). First, the raw recordings were filtered in broadband (0.1-100 $\mathrm{Hz}$ ) and downsampled to $1000 \mathrm{~Hz}$. Additionally, we applied a notch filter around the frequency of line noise from 48 to $52 \mathrm{~Hz}$. Noisy channels were removed based on visual inspection and spectra inspection, and bad time segments were cut out manually. After cleaning the data, the mean length of the recording in the eyes-closed condition was 493 seconds, the eyes-open condition was slightly shorter - 417 seconds. Data were re-referenced to a common average reference. Second, the spectrum of the signal from all electrodes was computed using Welch's method with $50 \%$ overlapping 10-sec windows and a corresponding frequency resolution of $0.1 \mathrm{~Hz}$. Alpha peak was determined as the maximum point in averaged spectrum within an 8-13 Hz frequency range. With the obtained value of peak frequency, oscillatory components of activity in the alpha band were extracted from broadband recording with multivariate Spatio-Spectral Decomposition (SSD; Nikulin et al., 2011). SSD extracts components that have maximal power at the desired frequency while simultaneously suppressing power in the neighbourhood range, thus increasing the signal-to-noise ratio. The frequency band near the alpha peak was chosen as $\pm 1 \mathrm{~Hz}$ from the determined individual peak, and flanking frequencies 
on both sides are from \pm 2 to $\pm 3 H z$ from the corners of the centre frequency. Obtained spatial filters were multiplied by minus one, if necessary, so that the maximal value of spatial filter weights was always positive. Further analysis was based on the first five SSD components, as they have the largest signal-to-noise ratio and in general smooth topography. Additionally, time courses of components were visually inspected for some residual high-amplitude noise, and if needed cleaned manually. Third, each component was band-pass filtered in the alpha range, which is $\pm 2 \mathrm{~Hz}$ around the alpha peak defined individually for each component, and for another signal $\left(V_{b s}\right)$ the raw data were low-pass filtered with a cut-off frequency of $3 \mathrm{~Hz}$. For that purpose, we utilized the Butterworth filter - order two for the alpha band and order four for low-frequency filtering (to control for the steepness of a filter response curve). The amplitude envelope of the bandpass signal $\left(V_{\text {alpha }}\right)$ was obtained using the Hilbert transform. Fourth, the amplitude envelope of alpha oscillations $\left(V_{\text {alpha }}\right)$ was divided into 20 percentile bins according to the magnitude, with the last bin containing the highest amplitude. The lowfrequency signal was sorted using the bins composition from the binning of values of $V_{\text {alpha }}$. Eventually, amplitudes in each bin were averaged to obtain a mean value of alpha envelope and corresponding low-frequency amplitude. Fifth, the relation between $V_{a l p h a}$ and $V_{b s}$ was estimated. In the previous research, the baseline-shift index (BSI) was computed as a slope of linear regression (Nikulin et al., 2010). However, in the current study, as the power was different in groups and conditions, we computed BSI as the Pearson correlation coefficient.

As for covariates, we computed the power ratio in the alpha band from the following formula $R_{a}=\frac{P_{a}}{P_{n}}$, where $P_{a}$ is mean spectral power around alpha peak $\pm 1 \mathrm{~Hz}$, and $P_{n}$ is mean spectral power in the flanking frequencies on both sides from \pm 2 to $\pm 3 \mathrm{~Hz}$ from the corners of the peak frequency. The power ratio in the low-frequency band was estimated as $R_{l f}=\frac{P_{l f}}{P_{n}}$, where $P_{l f}$ is mean spectral power from 0.1 to $3 \mathrm{~Hz}$, and $P_{n}$ is mean spectral power in the range from 0.1 to $7 \mathrm{~Hz}$.

\section{Statistical analysis}

To detect the difference between groups and conditions in absolute values of BSI, we used the ANOVA model (Python module statsmodels, anova_lm; Seabold and Perktold, 2010), with age and condition (eyes-closed vs eyes-open) as categorical independent variables, and with the power ratio in the alpha band and the power ratio in the low-frequency band as continuous independent variables. Both age and condition were treated as between-group variables. Despite the fact that the same individual's EEG was analysed, for each condition, we applied a distinct set of SSD filters. Therefore, the time courses after SSD filtering represent different sources or a superposition of different sources between eyes-closed and eyes-open conditions. The power ratio values were significantly skewed, and they were log-transformed for ANOVA testing. To determine how strong is the correlation between power ratio and BSI, we used the Pearson correlation coefficient.

\section{Simulation of networks with various degrees of spatial synchronization}

To examine possible effects of spatial synchronization on baseline shifts we simulated a time series of synchronous and asynchronous networks (Schaworonkow and Nikulin, 2019). Modelling was performed in MATLAB (R2018b, MathWorks). Each neuron in the network was modelled as a simple oscillator following the sinusoidal wave according to the formula:

$$
\mu(t)=A(t)\left[A_{\alpha} \sin \left(2 \pi f_{\alpha} t+\theta_{\alpha}\right)+A_{\beta} \sin \left(2 \pi f_{\beta} t+\theta_{\beta}\right)+r\right]
$$


where $A_{\alpha}, A_{\beta}$ are amplitudes of alpha and beta oscillations, respectively,

$f_{\alpha}, f_{\beta}$ - frequencies of alpha and beta oscillations,

$\theta_{\alpha}, \theta_{\beta}$ - phases of alpha and beta oscillations,

$r$ - OM of a signal from one neuron,

$A(t)$ - amplitude modulation.

Parameters in this model were set as follows $A_{\alpha}=1$ a.u., $A_{\beta}=0.25$ a.u., $f_{\alpha}=10 \mathrm{~Hz}$, $f_{\beta}=20 \mathrm{~Hz}, t=1 \mathrm{~s}, r=-0.4$ a.u. . Phases of each neuron were sampled from von Mises distribution with different concentration settings (Best and Fisher, 1979), using a MATLAB Toolbox CircStat (Berens, 2009). The probability density function of a random variable with von Mises distribution complies with equation $\phi(\theta)=\frac{1}{2 \pi I_{0}(\kappa)} e^{\kappa \cos \left(\theta-\mu_{0}\right)}$, where $I_{0}(\kappa)$ - is the modified Bessel function of the first kind and order $0, \mu_{0}$ - mean value, and $\kappa$ - the concentration parameter (Best and Fisher, 1979). Phases of beta were computed as shifted to $\pi / 4$ alpha phases to recreate the comb-like shape. Amplitude modulation $A(t)$ for the stimulus-induced oscillatory change was simulated as inverted Gaussian with varying widths of the left and right planks. Signals from each neuron were added together to produce a compound signal $X(t)=\sum_{i=1}^{N} \mu_{i}(t)+\epsilon$ (Schaworonkow and Nikulin, 2019), where $N=30000$ and $\epsilon$ - noise. The type of noise that's implemented was pink noise, as it exists in real EEG recordings. Inverse Fourier transform method was utilized to create the pink noise (van Driel et al., 2015). In addition to pink noise, Gaussian noise was also appended to the signal, which represented environmental noise (Telenczuk et al., 2010). Here $X(t)$ represents a single epoch response. The compound signal thus approximately represents a signal typically recorded with EEG/MEG. Further, to imitate the evoked response experimental procedure, the simulation was repeated for 100 epochs, and each time new phases have been sampled from von Mises distribution. The resulting signal was calculated according to the formula $E(t)=\sum_{j=1}^{K} X_{j}(t)$, where $K=100$ - number of epochs.

To design a synchronous and asynchronous network, we varied the concentration parameter $\kappa$ : for synchronous network $\kappa=1$, for asynchronous network (in which neurons have significant phase lags) $\kappa=10^{-6}$. The comparison between the two cases was made based on the waveform of a compound signal $X(t)$ and on the time course of the alpha envelope. For the extraction of the alpha envelope, the oscillation of each neuron was "filtered" in the alpha band resulting in a signal $\mu_{\alpha}(t)=A(t)\left[A_{\alpha} \sin \left(2 \pi f_{\alpha} t+\theta_{\alpha}\right)\right]$, which then was summed across all the neurons in the network $X_{\alpha}(t)=\sum_{i=1}^{N} \mu_{\alpha}^{i}(t)$ and Hilbert-transformed to extract the amplitude envelope over a single epoch.

\section{Data and code availability}

Data from the LEMON data set is publicly available at http : //fcon_1000.projects.nitrc.org/indi/retro/MPI_LEMON.html. For a complete description of the LEMON data see Babayan et al., 2019.

Custom Matlab functions for EEG analysis are available on Github http : //github.com/astudenova/bsi_matlab. 


\section{References}

Abeles, I. Y., \& Gomez-Ramirez, M. (2014). Impairments in background and event-related alpha-band oscillatory activity in patients with schizophrenia. PloS one, 9(3), e91720.

Ahmadi, M., Schoenfeld, M. A., Hillyard, S. A., \& Quiroga, R. Q. (2021). A simple metric to study the mechanisms generating event-related potentials. Journal of Neuroscience Methods, 109230.

Amit, R., Abeles, D., Carrasco, M., \& Yuval-Greenberg, S. (2019). Oculomotor inhibition reflects temporal expectations. Neuroimage, 184, 279-292.

Babayan, A., Erbey, M., Kumral, D., Reinelt, J. D., Reiter, A. M., Röbbig, J., Schaare, H. L., Uhlig, M., Anwander, A., Bazin, P.-L., et al. (2019). A mind-brain-body dataset of mri, eeg, cognition, emotion, and peripheral physiology in young and old adults. Scientific data, 6(1), 1-21.

Bannister, A. P. (2005). Inter-and intra-laminar connections of pyramidal cells in the neocortex. Neuroscience research, 53(2), 95-103.

Becker, R., Ritter, P., \& Villringer, A. (2008). Influence of ongoing alpha rhythm on the visual evoked potential. Neuroimage, 39(2), 707-716.

Bekkers, J. M. (2000). Properties of voltage-gated potassium currents in nucleated patches from large layer 5 cortical pyramidal neurons of the rat. The Journal of physiology, 525(3), 593-609.

Bender, S., Resch, F., Weisbrod, M., \& Oelkers-Ax, R. (2004). Specific task anticipation versus unspecific orienting reaction during early contingent negative variation. Clinical neurophysiology, 115(8), 1836-1845.

Benhassine, N., \& Berger, T. (2005). Homogeneous distribution of large-conductance calciumdependent potassium channels on soma and apical dendrite of rat neocortical layer 5 pyramidal neurons. European Journal of Neuroscience, 21(4), 914-926.

Berens, P. (2009). Circstat: A matlab toolbox for circular statistics. Journal of statistical software, 31(1), 1-21.

Best, D., \& Fisher, N. I. (1979). Efficient simulation of the von mises distribution. Journal of the Royal Statistical Society: Series C (Applied Statistics), 28(2), 152-157.

Blankertz, B., Acqualagna, L., Dähne, S., Haufe, S., Schultze-Kraft, M., Sturm, I., Ušćumlic, M., Wenzel, M. A., Curio, G., \& Müller, K.-R. (2016). The berlin brain-computer interface: Progress beyond communication and control. Frontiers in Neuroscience, 10, 530.

Boivin, J. R., \& Nedivi, E. (2018). Functional implications of inhibitory synapse placement on signal processing in pyramidal neuron dendrites. Current opinion in neurobiology, $51,16-22$.

Bullock, T., McClune, M., Achimowicz, J., Iragui-Madoz, V., Duckrow, R., \& Spencer, S. (1995). Eeg coherence has structure in the millimeter domain: Subdural and hippocampal recordings from epileptic patients. Electroencephalography and clinical Neurophysiology, 95(3), 161-177.

Bush, P. C., \& Sejnowski, T. J. (1993). Reduced compartmental models of neocortical pyramidal cells. Journal of neuroscience methods, 46(2), 159-166.

Buzsáki, G., Anastassiou, C. A., \& Koch, C. (2012). The origin of extracellular fields and currents - eeg, ecog, lfp and spikes. Nature reviews neuroscience, 13(6), 407-420.

Buzsáki, G., \& Draguhn, A. (2004). Neuronal oscillations in cortical networks. Science, 304(5679), 1926-1929. 
Dancey, C. P., \& Reidy, J. (2007). Statistics without maths for psychology. Pearson education, United Kingdom.

Demanuele, C., Broyd, S. J., Sonuga-Barke, E. J., \& James, C. (2013). Neuronal oscillations in the eeg under varying cognitive load: A comparative study between slow waves and faster oscillations. Clinical neurophysiology, 124(2), 247-262.

Destexhe, A., Contreras, D., \& Steriade, M. (1999). Cortically-induced coherence of a thalamicgenerated oscillation. Neuroscience, 92(2), 427-443.

Douglas, R. J., \& Martin, K. A. (2004). Neuronal circuits of the neocortex. Annu. Rev. Neurosci., 27, 419-451.

Elul, R. (1972). The genesis of the eeg. International review of neurobiology, 15, 227-272.

Ergenoglu, T., Demiralp, T., Bayraktaroglu, Z., Ergen, M., Beydagi, H., \& Uresin, Y. (2004). Alpha rhythm of the eeg modulates visual detection performance in humans. Cognitive brain research, 20(3), 376-383.

Fukuda, K., Mance, I., \& Vogel, E. K. (2015). $\alpha$ power modulation and event-related slow wave provide dissociable correlates of visual working memory. Journal of Neuroscience, 35(41), 14009-14016.

Funderud, I., Lindgren, M., Løvstad, M., Endestad, T., Voytek, B., Knight, R. T., \& Solbakk, A.-K. (2012). Differential go/nogo activity in both contingent negative variation and spectral power. PLoS One, 7(10), e48504.

Hald, L. A., Bastiaansen, M. C., \& Hagoort, P. (2006). Eeg theta and gamma responses to semantic violations in online sentence processing. Brain and language, 96(1), 90-105.

Hanslmayr, S., Klimesch, W., Sauseng, P., Gruber, W., Doppelmayr, M., Freunberger, R., Pecherstorfer, T., \& Birbaumer, N. (2007). Alpha phase reset contributes to the generation of erps. Cerebral Cortex, 17(1), 1-8.

Heimann, K. S., Uithol, S., Calbi, M., Umiltà, M. A., Guerra, M., \& Gallese, V. (2017). cuts in action": A high-density eeg study investigating the neural correlates of different editing techniques in film. Cognitive science, 41(6), 1555-1588.

Hong, X., Sun, J., Bengson, J. J., Mangun, G. R., \& Tong, S. (2015). Normal aging selectively diminishes alpha lateralization in visual spatial attention. NeuroImage, 106, 353-363.

Iemi, L., Busch, N. A., Laudini, A., Haegens, S., Samaha, J., Villringer, A., \& Nikulin, V. V. (2019). Multiple mechanisms link prestimulus neural oscillations to sensory responses. Elife, 8, e43620.

Iemi, L., Gwilliams, L., Samaha, J., Auksztulewicz, R., Cycowicz, Y. M., King, J.-R., Nikulin, V. V., Thesen, T., Doyle, W., Devinsky, O., et al. (2021). Spontaneous neural oscillations influence behavior and sensory representations by suppressing neuronal excitability. preprint at https://www.biorxiv.org/content/10.1101/2021.03.01.433450v1.abstract.

Ishii, R., Canuet, L., Aoki, Y., Hata, M., Iwase, M., Ikeda, S., Nishida, K., \& Ikeda, M. (2017). Healthy and pathological brain aging: From the perspective of oscillations, functional connectivity, and signal complexity. Neuropsychobiology, 75 (4), 151-161.

Jones, S. R., Pinto, D. J., Kaper, T. J., \& Kopell, N. (2000). Alpha-frequency rhythms desynchronize over long cortical distances: A modeling study. Journal of computational neuroscience, 9(3), 271-291.

Jones, S. R., Pritchett, D. L., Sikora, M. A., Stufflebeam, S. M., Hämäläinen, M., \& Moore, C. I. (2009). Quantitative analysis and biophysically realistic neural modeling of the meg mu rhythm: Rhythmogenesis and modulation of sensory-evoked responses. Journal of neurophysiology, 102(6), 3554-3572. 
Jones, S. R., Pritchett, D. L., Stufflebeam, S. M., Hämäläinen, M., \& Moore, C. I. (2007). Neural correlates of tactile detection: A combined magnetoencephalography and biophysically based computational modeling study. Journal of Neuroscience, 27(40), 1075110764 .

Kerr, C. E., Sacchet, M. D., Lazar, S. W., Moore, C. I., \& Jones, S. R. (2013). Mindfulness starts with the body: Somatosensory attention and top-down modulation of cortical alpha rhythms in mindfulness meditation. Frontiers in human neuroscience, \%, 12.

Lee, S., \& Jones, S. R. (2013). Distinguishing mechanisms of gamma frequency oscillations in human current source signals using a computational model of a laminar neocortical network. Frontiers in human neuroscience, 7, 869.

Makeig, S., Westerfield, M., Jung, T.-P., Enghoff, S., Townsend, J., Courchesne, E., \& Sejnowski, T. J. (2002). Dynamic brain sources of visual evoked responses. Science, 295(5555), 690-694.

Mäkinen, V., Tiitinen, H., \& May, P. (2005). Auditory event-related responses are generated independently of ongoing brain activity. NeuroImage, $24(4), 961-968$.

Mazaheri, A., \& Jensen, O. (2006). Posterior $\alpha$ activity is not phase-reset by visual stimuli. Proceedings of the National Academy of Sciences, 103(8), 2948-2952.

Mazaheri, A., \& Jensen, O. (2008). Asymmetric amplitude modulations of brain oscillations generate slow evoked responses. Journal of Neuroscience, 28(31), 7781-7787.

Monto, S., Palva, S., Voipio, J., \& Palva, J. M. (2008). Very slow eeg fluctuations predict the dynamics of stimulus detection and oscillation amplitudes in humans. Journal of Neuroscience, 28(33), 8268-8272.

Neymotin, S. A., Daniels, D. S., Caldwell, B., McDougal, R. A., Carnevale, N. T., Jas, M., Moore, C. I., Hines, M. L., Hämäläinen, M., \& Jones, S. R. (2020). Human neocortical neurosolver (hnn), a new software tool for interpreting the cellular and network origin of human meg/eeg data. Elife, 9, e51214.

Nikulin, V. V., Linkenkaer-Hansen, K., Nolte, G., \& Curio, G. (2010). Non-zero mean and asymmetry of neuronal oscillations have different implications for evoked responses. Clinical Neurophysiology, 121(2), 186-193.

Nikulin, V. V., Linkenkaer-Hansen, K., Nolte, G., Lemm, S., Müller, K. R., Ilmoniemi, R. J., \& Curio, G. (2007). A novel mechanism for evoked responses in the human brain. European Journal of Neuroscience, 25(10), 3146-3154.

Nikulin, V. V., Nolte, G., \& Curio, G. (2011). A novel method for reliable and fast extraction of neuronal eeg/meg oscillations on the basis of spatio-spectral decomposition. NeuroImage, 55(4), 1528-1535.

Osipova, D., Hermes, D., \& Jensen, O. (2008). Gamma power is phase-locked to posterior alpha activity. PloS one, 3(12), e3990.

Palva, S., \& Palva, J. M. (2007). New vistas for $\alpha$-frequency band oscillations. Trends in neurosciences, 30(4), 150-158.

Pfurtscheller, G., \& Da Silva, F. L. (1999). Event-related eeg/meg synchronization and desynchronization: Basic principles. Clinical neurophysiology, 110(11), 1842-1857.

Rawls, E., Miskovic, V., \& Lamm, C. (2020). Delta phase reset predicts conflict-related changes in p3 amplitude and behavior. Brain research, 1730, 146662.

Roberts, D. M., Fedota, J. R., Buzzell, G. A., Parasuraman, R., \& McDonald, C. G. (2014). Prestimulus oscillations in the alpha band of the eeg are modulated by the difficulty of feature discrimination and predict activation of a sensory discrimination process. Journal of cognitive neuroscience, 26(8), 1615-1628. 
Rossini, P. M., Rossi, S., Babiloni, C., \& Polich, J. (2007). Clinical neurophysiology of aging brain: From normal aging to neurodegeneration. Progress in neurobiology, 83(6), 375400.

Savers, B. M., Beagley, H., \& Henshall, W. (1974). The mechanism of auditory evoked eeg responses. Nature, $247(5441), 481-483$.

Schaworonkow, N., \& Nikulin, V. V. (2019). Spatial neuronal synchronization and the waveform of oscillations: Implications for eeg and meg. PLoS Computational Biology, 15(5), e1007055.

Schneider, J. M., \& Maguire, M. J. (2018). Identifying the relationship between oscillatory dynamics and event-related responses. International Journal of Psychophysiology, 133, $182-192$.

Schomer, D. L., \& Da Silva, F. L. (2010). Niedermeyer's electroencephalography: Basic principles, clinical applications, and related fields. Lippincott Williams \& Wilkins, Philadelphia.

Seabold, S., \& Perktold, J. (2010). Statsmodels: Econometric and statistical modeling with python. 9th Python in Science Conference.

Shah, A. S., Bressler, S. L., Knuth, K. H., Ding, M., Mehta, A. D., Ulbert, I., \& Schroeder, C. E. (2004). Neural dynamics and the fundamental mechanisms of event-related brain potentials. Cerebral cortex, $14(5), 476-483$.

Shibasaki, H., \& Hallett, M. (2006). What is the bereitschaftspotential? Clinical neurophysiology, 117(11), 2341-2356.

Spruston, N. (2008). Pyramidal neurons: Dendritic structure and synaptic integration. Nature Reviews Neuroscience, 9(3), 206-221.

Stephani, T., Waterstraat, G., Haufe, S., Curio, G., Villringer, A., \& Nikulin, V. V. (2020). Temporal signatures of criticality in human cortical excitability as probed by early somatosensory responses. Journal of Neuroscience, 40(34), 6572-6583.

Störmer, V. S., Feng, W., Martinez, A., McDonald, J. J., \& Hillyard, S. A. (2016). Salient, irrelevant sounds reflexively induce alpha rhythm desynchronization in parallel with slow potential shifts in visual cortex. Journal of cognitive neuroscience, 28(3), 433445 .

Telenczuk, B., Nikulin, V. V., \& Curio, G. (2010). Role of neuronal synchrony in the generation of evoked eeg/meg responses. Journal of neurophysiology, 104(6), 3557-3567.

Tzelepi, A., Bezerianos, T., \& Bodis-Wollner, I. (2000). Functional properties of sub-bands of oscillatory brain waves to pattern visual stimulation in man. Clinical Neurophysiology, $111(2), 259-269$.

van Driel, J., Cox, R., \& Cohen, M. X. (2015). Phase-clustering bias in phase-amplitude cross-frequency coupling and its removal. Journal of Neuroscience Methods, 254, 6072.

Wood, C. C., \& Allison, T. (1981). Interpretation of evoked potentials: A neurophysiological perspective. Canadian Journal of Psychology/Revue canadienne de psychologie, 35(2), 113.

Xia, J., Mazaheri, A., Segaert, K., Salmon, D. P., Harvey, D., Shapiro, K., Kutas, M., \& Olichney, J. M. (2020). Event-related potential and eeg oscillatory predictors of verbal memory in mild cognitive impairment. Brain communications, 2(2), fcaa213.

Yordanova, J., Kolev, V., \& Polich, J. (2001). P300 and alpha event-related desynchronization (erd). Psychophysiology, 38(1), 143-152.

Ziegler, D. A., Pritchett, D. L., Hosseini-Varnamkhasti, P., Corkin, S., Hämäläinen, M., Moore, C. I., \& Jones, S. R. (2010). Transformations in oscillatory activity and evoked re- 
sponses in primary somatosensory cortex in middle age: A combined computational neural modeling and meg study. Neuroimage, 52(3), 897-912.

\section{Contributions}

VVN and AV conceived the research; AAS and VVN conducted experiments and analyzed the results. All authors wrote and reviewed the manuscript.

\section{Competing interests}

The authors declare no competing financial interests. 